\section{Clinical evaluation of changes in cornea and tear film after surgery for trachomatous upper lid entropion}

P Monga, VP Gupta and U Dhaliwal
Department of

Ophthalmology, University

College of Medical Sciences and Guru Teg Bahadur

Hospital, Delhi, India

Correspondence:

U Dhaliwal, A-61,

Govindpuram,

Ghaziabad 201002,

UP, India

Tel: 91-120-2764747;

Fax: 91-11-22590495

E-mail: upreetdhaliwal@

yahoo.com

Received: 14 October 2006 Accepted in revised form: 30 January 2007

Published online: 2 March 2007

Proprietary interests: none Research funding: none

\begin{abstract}
Purpose To determine the effect of entropion surgery on corneal health in terms of surface epithelium, tear film stability, change in curvature and vision.

Methods Prospective study; included consecutive patients with trachomatous upper lid entropion undergoing corrective surgery. Corneal opacity and other conditions that reduced vision were recorded. Best-corrected visual acuity, corneal fluorescein staining, tear film break-up time, Schirmer I test, and keratometry were performed before and after surgery. Data were analysed using Student's $t$-test, $\chi^{2}$ test, one-way ANOVA, one-sample $t$-test, and logistic and multiple regression. Results Thirty-six female and 15 male patients with an average age of $59.1 \pm 10.65$ years were included. Lenticular and retinal causes resulted in blindness $(P=0.008)$, and low vision $(P=0.02)$, more often than entropion. At 90 days after surgery, vision improved by $0.55 \pm 1.48$ lines $(P=0.01)$. Superficial punctate staining of the cornea reduced by half over 1-15 days (average 6.96 \pm 4.99 ) after surgery, and completely in 1-90 days (average $26.15 \pm 17.49$ ). Tear film break-up time (range: 3-20 s preoperatively) improved significantly after surgery $(P=0.005)$ whereas Schirmer I (range: $10-35 \mathrm{~mm}$ preoperatively) and keratometry values showed insignificant change.

Conclusions Surgery for entropion results in healing of superficial keratopathy, improves tear film stability, and the realigned lid margin spreads tears evenly and efficiently, thus contributing to improved vision. These changes, taking place over 1-90 days, should be kept in mind when planning intraocular
\end{abstract}

surgery, keratoplasty, or keratorefractive procedures after entropion correction. Eye (2008) 22, 912-917; doi:10.1038/sj.eye.6702768; published online 2 March 2007

Keywords: corneal epithelium; entropion surgery; keratometry; tear film

\section{Introduction}

Trachoma, by virtue of its chronic inflammatory and cicatrizing effect on conjunctival epithelium, affects both aqueous and mucin secretion, resulting in an unstable tear film. ${ }^{1-3}$ Its blinding effect on the cornea is consequent not only to infiltration, but also to trichiasis causing recurrent corneal epithelopathy and infectious corneal ulcers, and to defective tears. ${ }^{2,45}$ Although some improvement in corneal opacification and vision has been reported following surgery to correct trichiasis or entropion a similar effect on tear film stability has not been reported earlier. ${ }^{4,5}$

Even in normal conditions, lid pressure has been shown to affect corneal shape. ${ }^{6,7}$ This effect can be accentuated in pathological lid conditions, and also after surgery to correct them, resulting in astigmatism and reduced vision. ${ }^{6,8-15}$ Although change in corneal curvature has been reported after correction of epiblepharon, congenital entropion, and ptosis, the effect after correction of trachomatous entropion has not previously been studied. ${ }^{11,14,16,17}$

The aim of this study was to determine the effect of entropion surgery on corneal health in terms of surface epithelium, tear film stability, change in curvature, and vision. Changes in these parameters after surgery for entropion 
could affect vision in spectacle and contact lens wearers, and impact subsequent interventions like keratoplasty, keratorefractive, and intraocular surgery.

\section{Materials and methods}

Consecutive patients with trachomatous upper lid entropion undergoing corrective surgery over a 1-year period were prospectively examined after informed consent and Institutional Ethical Committee clearance. The diagnosis of trachoma was on the basis of clinical criteria recommended by the World Health Organization. ${ }^{18}$

Best-corrected visual acuity (BCVA), location of corneal opacity (peripheral, other than trachomatous pannus, or central), and any other conditions capable of reducing vision were recorded in these patients. Visual loss was graded, according to the definition given by $\mathrm{WHO}$, into low vision (BCVA $<6 / 18$ but $\geqslant 3 / 60$ in the better eye) and blindness (BCVA $<3 / 60$ in the better eye). ${ }^{19}$ Corneal fluorescein staining was carried out and the area of staining was recorded diagrammatically by the same observer (PKM) in all cases. It was graded from 0 to 2 , where $0=$ no stain; $1=$ superficial punctate staining of $\leqslant$ half of the corneal surface; and $2=$ more than half of the surface took up the stain. To reduce bias, the observer was masked to previous corneal drawings at subsequent follow-up visits. None of the patients received lubricating eye drops before or after surgery. Tear film break up time (BUT), Schirmer I test (using Whatman number 41 filter paper), and keratometry in two dominant corneal meridia (using Bausch \& Lomb type keratometer) were performed and the results recorded.

The original cohort included 90 consecutive patients with moderate to severe (without lid gap) trachomatous entropion. These patients were part of a study investigating the efficacy of three surgical procedures in correcting trachomatous entropion. ${ }^{20}$ Kemp and Collin's ${ }^{21}$ grading of moderate and severe entropion was used. The surgical procedure was allocated by computer-generated randomization and included terminal tarsal rotation after transverse tarsotomy (Kettesy's procedure), ${ }^{22}$ tarsal rotation with tarsoconjunctival advancement (Collin's modification of Trabut's procedure), ${ }^{23}$ and anterior lamellar repositioning with lid margin split and wedge resection of tarsus. ${ }^{19}$ These are routinely performed in this hospital and are established procedures in the correction of moderate to severe (without lid gap) trachomatous entropion. ${ }^{19}$ The surgical results, including intraoperative and postoperative complications, were comparable for the three procedures. These results, including preoperative and postoperative photographs, have been published earlier. ${ }^{20}$ This was the second arm of the study and included only those patients where preoperative keratometry was possible; thus patients with dense corneal opacity and severe dry eye were excluded from analysis.

After each procedure, the eye was bandaged for $24 \mathrm{~h}$ with ciprofloxacin $0.3 \%$ eye ointment. Patients were prescribed oral ciprofloxacin (500 $\mathrm{mg}$ twice a day for 5 days) and ibuprofen ( $400 \mathrm{mg}$ thrice a day for 3 days). Ciprofloxacin $0.3 \%$ eye drops were instilled starting at least 3 days before surgery and continued up to the 15th postoperative day.

Patients were followed up on postoperative day 1 , and then weekly for corneal fluorescein staining, and on day 90 for BCVA, tear film break-up time, Schirmer I test and keratometry in two dominant meridia.

Data were subjected to statistical analysis. Unpaired and paired Student's $t$-test, $\chi^{2}$ and Fisher's exact tests at the $5 \%$ level, one-way ANOVA, one-sample $t$-test, and logistic and multiple regression was used.

\section{Results}

The study included 51 patients (51 eyes) with age ranging from 32 to 75 years (average age $59.1 \pm 10.65$ ). There were 36 female and 15 male patients. The duration of entropion varied from 1 month to 15 years (average $56.7 \pm 52.12$ months). Entropion was moderately severe in 38 eyes and severe, without lid gap on gentle closure, in 13 eyes. Terminal tarsal rotation after transverse tarsotomy (Kettesy's procedure) ${ }^{22}$ and tarsal rotation with tarsoconjunctival advancement (Collin's modification of Trabut's procedure $)^{23}$ were performed in 18 eyes each, whereas anterior lamellar repositioning with lid margin split and wedge resection of tarsus ${ }^{19}$ was performed in 15 eyes.

Preoperative BCVA and causes for poor vision are shown in Table 1. Lenticular and retinal causes were responsible for blindness $(P=0.008)$ and for low vision $(P=0.02)$, significantly more often than entropion-related causes (Fisher's exact test). Trachomatous corneal opacities, other than pannus, were seen in 12 patients, located peripherally in eight eyes and centrally in five eyes. The presence of opacity $(P=0.08)$, or its location $(P=0.38)$ did not significantly affect preoperative vision $\left(\chi^{2}\right.$ test). On performing logistic regression, taking corneal opacity as the dependent variable, opacities were not related to patient's gender, age, or duration of entropion. Tear film BUT, however, was significantly lower in patients with opacities $(P=0.01 ; \beta$-coefficient -0.199 ; odds ratio $0.819 ; 95 \%$ confidence interval 0.703-0.955).

At 90 days after surgical correction of entropion, BCVA improved in the cohort by $0.55 \pm 1.48$ lines $(P=0.01$, 
one-sample $t$-test). However, BCVA worsened in five patients $(9.8 \%$ ) by $1-5$ lines (average $2.0 \pm 1.73$ ) and was invariably due to progression of cataract $(n=4)$ or posterior capsular opacification $(n=1)$. BCVA remained stable in $22(43.1 \%)$ and improved in $24(47.0 \%)$ patients.

Fluorescein staining of the cornea revealed superficial punctate staining in all patients, involving half or less of the corneal surface in 17 eyes, and greater than half in 34 eyes. There were comparable number of patients with lesser or greater corneal staining in the three procedure groups ( $P=0.75 ; \chi^{2}$ test). On performing logistic regression, taking staining as the dependent variable, there was no relationship with vision $<6 / 18$, age of patient, or duration of entropion. However, greater area of corneal staining was significantly related to lower preoperative BUT values $(P=0.03 ; \beta$-coefficient -0.181 ; odds ratio $0.834 ; 95 \%$ confidence interval 0.707-0.985). The area of staining reduced by half over 1-15 days (average $6.96 \pm 4.99$ ) after surgery, whereas complete resolution was seen in 1-90 days (average $26.15 \pm 17.49$ ). On multiple regression analysis, taking speed of resolution as the dependent variable, there was no relation with age, gender, duration, grade of entropion, area of staining, or preoperative BUT values $(P>0.05$ each).

Preoperative tear film BUT ranged from 3 to $20 \mathrm{~s}$, whereas Schirmer I test values varied from 10 to $35 \mathrm{~mm}$ in $5 \mathrm{~min}$. Preoperatively, no patient had signs of overt dry eyes. Average BUT values improved significantly after surgery (Table 2). Although average Schirmer I values reduced after intervention, the difference was not statistically significant.

Preoperative and 90-day postoperative values of keratometry in the horizontal and vertical meridia are shown in Table 2. Average horizontal and vertical corneal curvatures steepened after surgery. Thus, corneal astigmatism, that was with the rule, averaging $0.24 \pm 2.64$ diopters preoperatively, changed to $0.34 \pm 2.33$, 90 days after surgery ( $P=0.61$; paired Student's $t$-test). Although vertical curvature seemed to increase more than the horizontal, the difference was not statistically significant.

The three procedures were compared for their effect on corneal epithelial healing, tear parameters, and keratometry values (Table 3 ). The degree of corneal

Table 1 Causes of decreased vision in 51 patients with trachomatous upper lid entropion

\begin{tabular}{|c|c|c|c|c|c|c|c|c|c|}
\hline \multirow{3}{*}{$\begin{array}{l}\text { Pre-operative } \\
\text { BCVA }\end{array}$} & \multirow{3}{*}{$\begin{array}{c}\text { Total } \\
\text { No. }(\%)\end{array}$} & \multicolumn{8}{|c|}{ Cause of decreased vision No. (\%) } \\
\hline & & \multicolumn{3}{|c|}{ Related to entropion alone } & \multicolumn{3}{|c|}{ Unrelated to entropion } & \multicolumn{2}{|c|}{ More than one cause } \\
\hline & & $\begin{array}{l}\text { SPK } \\
\text { alone }\end{array}$ & PCO & $\mathrm{CCO}$ & Cataract & $\begin{array}{c}\text { Posterior capsular } \\
\text { opacity }\end{array}$ & $\begin{array}{l}\text { Retinal } \\
\text { disease }^{\mathrm{a}}\end{array}$ & $\begin{array}{l}\text { Cataract } \\
\text { and PCO }\end{array}$ & $\begin{array}{l}\text { Cataract } \\
\text { and } \mathrm{CCO}\end{array}$ \\
\hline $6 / 6-6 / 18$ & 16 (31.4) & 9 & 0 & 1 & 2 & 4 & 0 & 0 & 0 \\
\hline $6 / 24-\geqslant 3 / 60$ & $23(45.1)$ & 4 & 1 & 1 & 4 & 3 & 4 & 4 & 2 \\
\hline$<3 / 60$ & $12(23.5)$ & 0 & 0 & 0 & 5 & 0 & 4 & 2 & 1 \\
\hline Total & 51 & 13 (25.5) & 1 (1.9) & $2(3.9)$ & 11 (21.6) & 7 (13.7) & 8 (15.7) & 6 (11.8) & $3(5.9)$ \\
\hline
\end{tabular}

Abbreviations: BCVA, best corrected visual acuity; CCO, central corneal opacity; PCO, peripheral corneal opacity ; SPK, superficial punctate keratitis. a Age-related macular degeneration $=3$, cystoid macular oedema $=2$, vitreous haemorrhage $=1$, central retinal vein occlusion $=1$, degenerative myopia $=1$.

Table 2 Change in tear film break up time, Schirmer I and keratometry values after corrective surgery in 51 patients with trachomatous upper lid entropion

\begin{tabular}{|c|c|c|c|c|c|}
\hline \multirow[t]{2}{*}{ Parameter } & \multicolumn{2}{|c|}{ Preoperative value } & \multicolumn{2}{|c|}{ Postoperative value } & \multirow[t]{2}{*}{ P-value paired Student's t-test } \\
\hline & Average & $S D$ & Average & $S D$ & \\
\hline Tear film BUT (s) & 9.8 & 5.02 & 11.4 & 2.77 & 0.005 \\
\hline Schirmer I (mm) & 21.8 & 8.29 & 20.9 & 6.64 & 0.54 \\
\hline Horizontal keratometry (D) & 44.1 & 2.33 & 44.2 & 2.39 & 0.35 \\
\hline Vertical keratometry $(\mathrm{D})$ & 44.3 & 2.57 & 44.5 & 2.26 & 0.23 \\
\hline Change in horizontal keratometry after surgery & \multicolumn{4}{|c|}{$0.12 \pm 0.89$} & 0.59 \\
\hline Change in vertical keratometry after surgery & \multicolumn{4}{|c|}{$0.22 \pm 1.30$} & \\
\hline
\end{tabular}

Abbreviations: BUT, break up time; SD, standard deviation. 
Table 3 Effect of type of corrective procedure on corneal epithelial healing, tear parameters, and keratometry values

\begin{tabular}{|c|c|c|c|c|c|c|c|}
\hline \multirow[t]{2}{*}{ Parameter } & \multicolumn{2}{|c|}{ Procedure I } & \multicolumn{2}{|c|}{ Procedure II } & \multicolumn{2}{|c|}{ Procedure III } & \multirow[t]{2}{*}{ P-value one-way ANOVA } \\
\hline & Average & $S D$ & Average & $S D$ & Average & $S D$ & \\
\hline Speed of resolution of corneal staining (days) & 18.2 & 7.98 & 33.4 & 21.47 & 27.0 & 15.21 & 0.02 \\
\hline Preoperative BUT (s) & 9.3 & 3.69 & 9.7 & 5.06 & 10.6 & 6.42 & 0.75 \\
\hline Postoperative change in BUT & 2.0 & 2.83 & 1.8 & 4.07 & 0.9 & 4.69 & 0.68 \\
\hline Preoperative Schirmer I (mm) & 19.9 & 6.14 & 21.5 & 9.69 & 24.3 & 8.66 & 0.19 \\
\hline Postoperative change in Schirmer I & 1.5 & 8.73 & -0.6 & 10.99 & -3.93 & 9.46 & 0.29 \\
\hline Change in Horizontal keratometry (D) & 0.6 & 0.89 & 0.4 & 1.34 & -0.5 & 1.45 & 0.03 \\
\hline Change in Vertical keratometry (D ) & 0.1 & 0.67 & -0.06 & 0.73 & 0.3 & 1.24 & 0.49 \\
\hline
\end{tabular}

Procedure I: Terminal tarsal rotation after transverse tarsotomy (Kettesy's procedure). ${ }^{22}$

Procedure II: Tarsal rotation with tarsoconjunctival advancement (Collin's modification of Trabut's procedure). ${ }^{23}$

Procedure III: Anterior lamellar repositioning with lid margin split and wedge resection of tarsus. ${ }^{19}$

staining was comparable for the three procedure groups, involving half or less of the corneal surface in five, seven, and five eyes, and greater than half in 13,11 , and 10 eyes, respectively ( $P=0.78 ; \chi^{2}$ test). Terminal tarsal rotation after transverse tarsotomy resulted in significantly faster healing of the corneal epithelium, whereas anterior lamellar repositioning with lid margin split and wedge resection of tarsus resulted in a relative flattening of the horizontal meridian of the cornea compared to the other two procedures.

\section{Discussion}

Entropion and trichiasis due to trachoma are an important cause of corneal blindness worldwide. ${ }^{24}$ However, in this study, significantly more patients with entropion had low vision or blindness due to lenticular causes, rather than corneal. This could be taken to mean that trachoma, although still prevalent, is losing its blinding potential in this part of the world. However, low vision and blindness in trachoma are difficult to measure adequately. ${ }^{25}$ Moreover, this was a hospital-based study, and we excluded patients with dense corneal opacity where keratometry was not possible. Thus, the data on blindness are likely to be biased and do not reflect the prevalence of blindness due to trachoma in the community. Vision improved after surgery for entropion in $47.0 \%(24 / 51)$ patients, even when patients had lenticular causes of reduced vision. This improvement is likely to have occurred from a combination of factors that resulted from correction of entropion. Improvement in the status of corneal epithelium and the tear film, as deduced from the temporal improvement in corneal staining and tear film BUT, were probably both responsible. In addition, reduction in reflex lacrimation may have contributed. The change in vision in our patients is likely to persist, unlike that reported after ptosis surgery, where transient changes in corneal curvature were temporarily responsible for change in vision. ${ }^{11,17}$

Trichiatic cilia, rubbing on the cornea, produce numerous superficial punctate erosions. Grossly, these are visible as a hazy cornea, but are clearly delineated on staining with fluorescein. In our patients, these erosions healed completely after correction of entropion regardless of age, gender, duration or grade of entropion, area of staining, or type of surgical procedure. This suggests that appropriate surgical correction of entropion is adequate to heal the epithelium in all cases. Even though patients with larger areas of fluorescein staining had lower BUT values, the speed of resolution was comparable to those with higher BUT values. Final resolution took on an average 30 days, and never more than 90 days. This fact must be kept in mind when planning any other procedure on the cornea after entropion surgery. Intraocular surgeries, keratoplasty, or keratorefractive procedures should be deferred for at least 30, but preferably 90 days after correction of entropion. Abnormal tear film stability is known to jeopardize the results of such procedures. ${ }^{26-28}$ Even though preoperative BUT values were comparable in the three procedure groups, epithelial healing was significantly faster following terminal tarsal rotation after transverse tarsotomy. The reason for this is not immediately clear. This procedure takes the least time to perform, and involves the least surgical dissection. ${ }^{20}$ Possibly, these factors lead to faster healing of the realigned lid margin and, consequently, the corneal epithelium.

Tear film BUT reflects the quality and stability of the tear film. Corneal epithelial diseases may be accompanied by some tear film instability due to superficial epithelial abnormalities, even when the tears are otherwise normal. ${ }^{29}$ Trachoma is known to cause conjunctival scarring leading to tear film abnormality. ${ }^{3}$ In this study, BUT values varied between 3 and $20 \mathrm{~s}$, but no patient had drying of conjunctiva or cornea; low BUT 
may have been compensated by greater than average Schirmer I values. No patient received lubricating eye drops after surgery. Thus, improvement in BUT after surgery is likely to be owing to improved corneal and conjunctival epithelial integrity, directly related to removal of aggravating factors (entropion or trichiasis). Good apposition of the realigned lid margin to the eyeball may have helped by ensuring that the tear film was spread evenly and efficiently.

Mean wetting of Schirmer strips during the Schirmer I test, although statistically not significant, decreased postoperatively. By surgically correcting entropion, the irritative component of the disease was decreased, leading to decreased reflex lacrimation and lower Schirmer I readings. The basal secretion test was not performed in this study. It might have been a good choice as it may indicate how the basal secretors are impacted after surgery to correct entropion.

Corneal curvature did not change significantly after surgery in our study, suggesting minimal or no effect of trachomatous upper lid entropion on corneal curvature. Similar results have been described after correction of epiblepharon, using retrospective data on refractive errors. ${ }^{14}$ However, some authors have used computerized corneal topography or videokeratoscopy to demonstrate change in corneal curvature in ptosis, ${ }^{13}$ meibomian cyst, ${ }^{6}$ and chalazion, ${ }^{9}$ and after blepharoplasty or surgery for ptosis. ${ }^{11,16,17}$ Some suggest that changes in corneal curvature may be missed on manifest refraction or keratometry, but show up on topography. ${ }^{13}$ A study using computerized corneal topography may reveal whether entropion surgery significantly changes corneal curvature. However, when individual procedures were considered, compared to the other two procedures, anterior lamellar repositioning with lid margin split and wedge resection of tarsus resulted in flattening of the horizontal meridian of the cornea. Although the other two procedures rotate the marginal tarsus by 90 or $180^{\circ}$, this procedure splits the lid margin and rotates the lash bearing anterior lamina alone. ${ }^{20}$ The released posterior lid margin may then be free to exert pressure on the cornea, resulting in changes in curvature. The proposed mechanism is purely speculative and needs to be corroborated in a larger number of cases.

This study demonstrates that surgery for entropion results in healing of superficial keratopathy, and improves tear film stability in patients with trachomatous upper lid entropion, contributing to improved vision. These changes take place over a period ranging from 1 to 90 days. One of the limitations of this study is that we did not evaluate the study patients for any additional ocular surgical interventions that they may have undergone after the study concluded.
Nevertheless, this time lag should be kept in mind when planning intraocular surgery, keratoplasty, or keratorefractive procedures after entropion correction.

\section{References}

1 Aksunger A, Unlu K, Karakas N, Nergiz Y, Celik Y. Impression cytology, tear film break up, and Schirmer test in patients with inactive trachoma. Jpn J Ophthalmol 1997; 41(5): 305-307.

2 Goldstein M, Loewenstein A, Rosner M, Lipshitz I, Lazar M. Phototherapeutic keratectomy in the treatment of corneal scarring from trachoma. J Refract Corneal Surg 1994; 10(2): S290-S292.

3 Moudgil SS, Singh M, Parmar IP, Khurana AK. Study of tear film flow and break up time (BUT) in trachoma. Acta Ophthalmol (Copenhagen) 1986; 64(3): 295-297.

4 Burton MJ, Bowman RJ, Faal H, Aryee EA, Ikumapayi UN, Alexander ND et al. Long term outcome of trichiasis surgery in the Gambia. Br J Ophthalmol 2005; 89(5): 575-579.

5 Reacher MH, Munoz B, Alghassany A, Daar AS, Elbualy M, Taylor HR. A controlled trial of surgery for trachomatous trichiasis of the upper lid. Arch Ophthalmol 1992; 110(5): 667-674.

6 Patel S. Changes in corneal topography and tear film stability due to a single Meibomian cyst. Am J Optom Physiol Opt 1987; 64(7): 528-530.

7 Lieberman DM, Grierson JW. The lids influence on corneal shape. Cornea 2000; 19(3): 336-342.

8 Cuttone JM, Durso F, Miller M, Evans LS. The relationship between soft tissue anomalies around the orbit and globe and astigmatic refractive errors: a preliminary report. J Pediatr Ophthalmol Strabismus 1980; 17(1): 29-36.

9 Santa Cruz CS, Culotta T, Cohen EJ, Rapuano CJ. Chalazion induced hyperopia as a cause of decreased vision. Ophthalmic Surg Lasers 1997; 28(8): 683-684.

10 Khwarg SI, Lee YJ. Epiblepharon of the lower lid: classification and association with astigmatism. Korean J Ophthalmol 1997; 11(2): 111-117.

11 Holck DE, Dutton JJ, Wehrly SR. Changes in astigmatism after ptosis surgery measured by corneal topography. Ophthalmol Plast Reconstr Surg 1998; 14(3): 151-158.

12 Goldhahn A, Schrom T, Berghaus A, Krause A, Duncker G. Corneal astigmatism as a special complication after lid loading in patients with lagophthalmos. Ophthalmologe 1999; 96(8): 494-497.

13 Kim T, Khosla-Gupta B, Debacker C. Blepharoptosisinduced keratoconus. Am J Ophthalmol 2000; 130(2): 232-234.

14 Yang SW, Choi WC, Kim SY. Refractive changes of congenital entropion and epiblepharon on surgical correction. Korean J Ophthalmol 2001; 15(1): 32-37.

15 Markomanolakis MM, Kymionis GD, Aslanides IM, Astyrakakis N, Pallikaris IG. Induced videokeratography alterations in patients with excessive meibomian secretions. Cornea 2005; 24(1): 16-19.

16 Brown MS, Siegel IM, Lisman RD. Prospective analysis of changes in corneal topography after upper eyelid surgery. Ophthalmol Plast Reconstr Surg 1999; 15(6): 378-383.

17 Kumar S, Chaudhuri Z, Chauhan D. Clinical evaluation of refractive changes following brow suspension surgery in pediatric patients with congenital blepharoptosis. Ophthalmic Surg Lasers Imaging 2005; 36(3): 217-227. 
18 Dawson CR, Jones BR, Tarizzo ML. Guide to Trachoma Control. World Health Organization: Geneva, 1981, pp 12-20.

19 World Health Organization. International Statistical Classification of Diseases and Related Health Problems, 10th revision: Version for 2006. World Health Organization: Geneva, 2006. Availiable at http://www.who.int/ classifications/apps/icd/icd 10 online/. Accessed February 2007.

20 Dhaliwal U, Monga PK, Gupta VP. Comparison of three surgical procedures of differing complexity in the correction of trachomatous upper lid entropion: a prospective study. Orbit 2004; 23(4): 227-236.

21 Kemp EG, Collin JRO. Surgical management of upper lid entropion. Br J Ophthalmol 1986; 70: 575-579.

22 Kettesy A. On genesis and operation of the cicatricial (trachomatous) entropion of the upper lid. Br J Ophthalmol 1948; 32: 419-423.

23 Collin JRO. Entropion and trichiasis. In: Collin JRO (ed). A Manual of Systematic Eyelid Surgery, 2nd edn. Butterworth and Heinemann: Oxford, 1989 pp 7-26.
24 Resnikoff S, Pascolini D, Etya'ale D, Kocur I, Pararajasegaram R, Pokharel GP et al. Global data on visual impairment in the year 2002. Bull WHO 2004; 82(11): 844-851.

25 Consultation on development of standards for characterization of vision loss and visual functioning. Availiable at http://whqlibdoc.who.int/hq/2003/ WHO_PBL_03.91.pdf. Accessed February 2007.

26 Paton $\mathrm{D}$. The prognosis of penetrating keratoplasty based upon corneal morphology. Ophthalmic Surg 1976; 7(3): 36-45.

27 Feng CM, Chen JO, Li YP. Factors of graft failure after chemical corneal injury. Zhonghua Yan Ke Za Zhi 1994; 30(4): 277-279.

28 Albietz JM, Lenton LM. Management of the ocular surface and tear film before, during, and after laser in situ keratomileusis. J Refract Surg 2004; 20(1): $62-71$.

29 Norihiko Y, Hiroshi S, Shigeru K. Direct observation of tear film stability on a damaged corneal epithelium [letter]. $\mathrm{Br} \mathrm{J}$ Ophthalmol 1998; 82: 1090. 